Schwerpunkt

Gastroenterologe 2021 · 16:179-185

https://doi.org/10.1007/s11377-021-00520-8

Angenommen: 10. März 2021

Online publiziert: 8. April 2021

๑ Der/die Autor(en) 2021

\section{Redaktion}

C. Trautwein, Aachen

J. Trebicka, Frankfurt am Main

Für den Tod von Patienten mit Leberzirrhose ist die akute Dekompensation (AD) ausschlaggebend, wobei das Zusammenspiel hepatischer Dekompensation mit extrahepatischer Organdysfunktion und -versagen maßgeblich ist. Die $A D$ weist verschiedene klinische Verläufe auf: von der stabilen dekompensierten Zirrhose über die instabile dekompensierte Zirrhose, das prä-akut-auf-chronische Leberversagen, bis hin zum akut-aufchronischen Leberversagen, das ein Syndrom mit Organversagen ist.

Die $\mathrm{AD}$ und ihre schwerste Form, das akut-auf-chronische Leberversagen (ACLF), sind die häufigste Todesursache bei der Leberzirrhose [1]. Da sie weltweit jährlich mehr als 14 Mio. krankheitsbereinigte Lebensjahre kostet, wird sie mittlerweile zu den führenden Problemen im Gesundheitswesen gerechnet. Die klinische Einschätzung und Risikobewertung dieser Patienten spielen eine große Rolle $[2,3]$.

\section{》) Die Schwere der AD korreliert mit dem Ausmaß der systemischen Inflammation}

Die wichtigsten Auslöser der AD sind nachgewiesene bakterielle Infektionen, die schwere alkoholische Hepatitis, gastrointestinale (GI-)Blutungen mit Schock sowie die toxische Enzephalopathie [4].

P. G. Ferstl · M. Schulz - J. Trebicka

Translationale Hepatologie, Klinik für Innere Medizin I, Goethe-Universität Frankfurt, Frankfurt am Main, Deutschland

\title{
Akute Dekompensation und akut-auf-chronisches Leberversagen
}

\section{Neues zur den Verlaufsformen der dekompensierten Leberzirrhose}

Der Beginn wie auch die Schwere der AD korrelieren mit dem Ausmaß der systemischen Inflammation (SI; $[4,5]$ ). Unter den verschiedenen Stadien der AD ist das Prä-ACLF der Zustand, der dem ACLF vorausgeht [6]. Treten Auslöser mit begleitendem Organversagen oder mehrere Auslöser gleichzeitig auf, haben AD und ACLF einen schwereren Verlauf [4]. Das ACLF wird mittlerweile als die schwerste Form der AD betrachtet, aber ihre klinischen Erscheinungsbilder sowie die inflammatorische „Visitenkarte“ unterscheiden sich maßgeblich. Die SI ist das gemeinsame Kennzeichen von AD und ACLF.

\section{Akute Dekompensation}

\section{Pathogenese}

Die portale Hypertension (PHT) ist ein zentraler Faktor in der Pathogenese der $\mathrm{AD}$ und des ACLF. Sie ist definiert als ein Zustand erhöhten hydrostatischen Drucks in der Portalvene und führt zur reflektorischen Dilatation splanchnischer Arteriolen und auch peripherer Arterien. Dies begünstigt durch ein vermindertes effektives arterielles Blutvolumen sowie die zirrhotische Kardiomyopathie die Entstehung des ACLF [5]. Ferner verursacht die PHT sekundär eine Schädigung der Darmbarriere mit konsekutiver bakterieller Translokation und Ausbildung von SI [7]. Die bakterielle Translokation tritt nur bei zirrhotischer PHT, nicht aber bei akuter bzw. nichtzirrhotischer PHT auf. Es ist daher bemerkenswert, dass die SI durch den transjugulären intrahepatischen portosystemischen Shunt (TIPS) reduziert wird, was u.a. ein Hinweis auf die Schlüsselrolle der PHT bei der Entstehung der SI ist [8, 9].

\section{》) Die PHT hat bei der Entstehung der systemischen Inflammation eine Schlüsselrolle}

Die SI kann laborchemisch durch die Leukozyten und das C-reaktive Protein abgebildet werden [6]. Doch es existiert eine Vielzahl weiterer potenzieller Biomarker der SI, die sensitiver und/oder spezifischer für die Entwicklung eines ACLF sind. So sind erhöhte Spiegel von Interleukin(IL)-6, IL-1RA und humanes Serumalbumin (HNA) 2 in AD-Patienten unabhängig mit der Entwicklung eines ACLF innerhalb von 28 Tagen assoziiert [3]. Das IL-1 $\alpha$, IL-1 $\beta$, Plasmarenin (PRC) und Copeptin (PCC) sowie weitere Zytokine sind zusätzliche Inflammationsmarker der AD, die eng mit der Ausbildung eines ACLF verbunden sind [10, 11]. Die SI ist beim ACLF deutlich ausgeprägter als in seinen Vorstufen stabile dekompensierte Zirrhose (SDC), instabile dekompensierte Zirrhose (UDC) und Prä-ACLF [10]. Ist die SI stark genug, um einen Endorganschaden auszulösen, kann sie ein ACLF triggern [3, 4].

Die SI und die AD prädisponieren den Organismus außerdem für „hyper- 


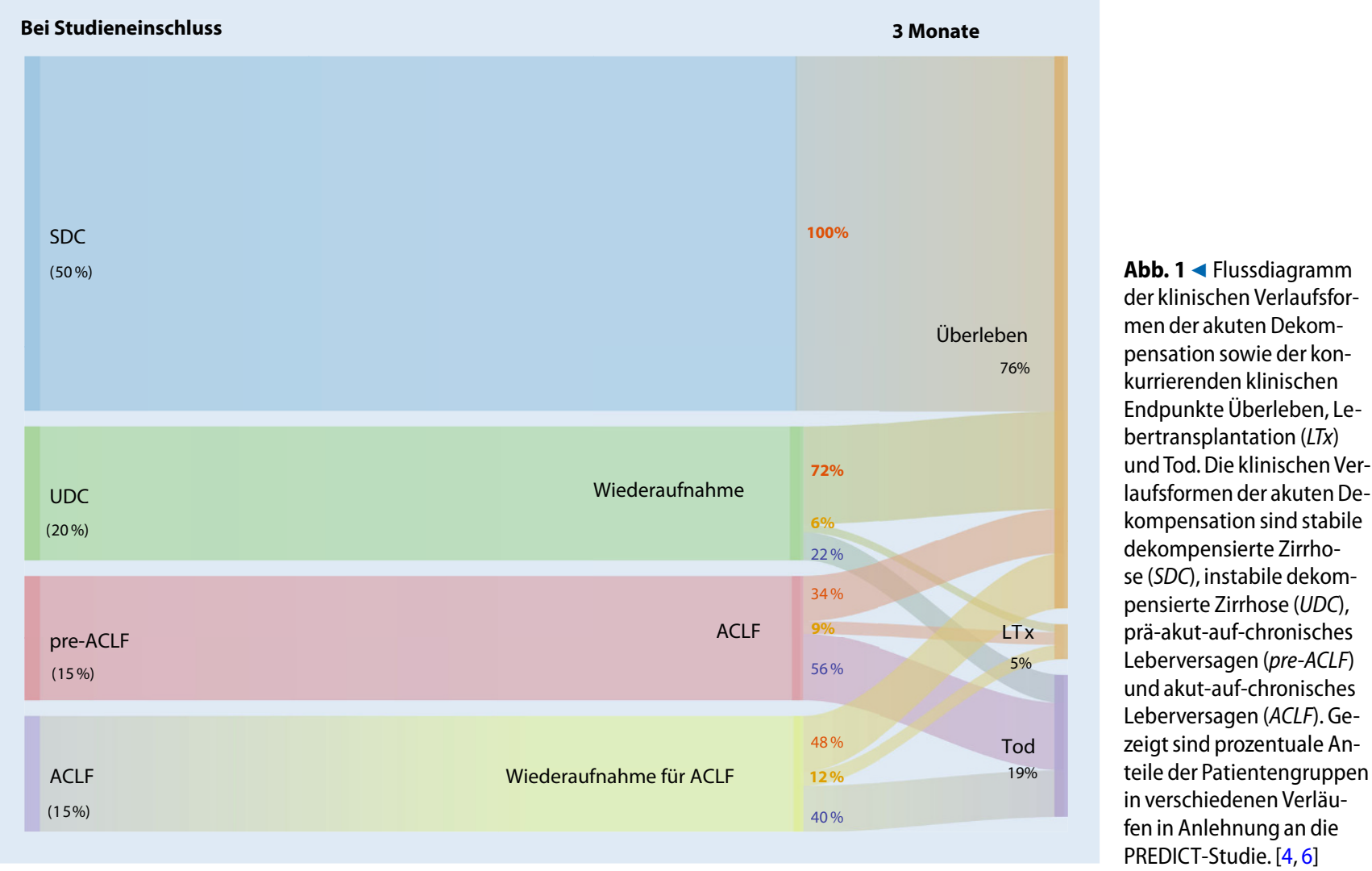

metabolische“ Zustände, in denen die Grundnährstoffe Glukose, Aminosäuren und Fettsäuren vorzugsweise Immunzellen mit einem hohen Energiebedarf bereitgestellt werden [12]. Die Deprivation von Nährstoffen in Verbindung mit inflammatorischen Stimuli kann letztlich Endorganversagen in Niere, Herz und Lunge und somit auch ein ACLF auslösen [13]. Inflammatorische Lipidmediatoren wie Leukotrien E4 $\left(\mathrm{LTE}_{4}\right)$ tragen zur SI bei und beeinträchtigen auch die bakterizide Aktivität neutrophiler Granulozyten [12]. Da beim ACLF eine unverhältnismäßig hohe Ausschüttung humoraler Marker beobachtet wird und gleichzeitig bakterielle Infektionen mit einer extrem hohen Rate auftreten, wird dem zellulären Immunsystem beim ACLF ein dysfunktionaler oder sogar paralysierter Zustand attestiert [14].

\section{Auslöserereignisse}

Ein Dekompensationsereignis wird als das Auftreten eines der folgenden $4 \mathrm{Zu}$ stände - oder deren Kombination - bezeichnet: Aszites, GI-Blutungen, hepa- tische Enzephalopathie (HE) und/oder bakterielle Infektion [15]. Diese Ereignisse komplizieren den klinischen Verlauf der Leberzirrhose in einer unvorhersehbaren Weise und treten häufig wiederkehrend auf. So ist die GI-Blutung signifikant mit der Entstehung der spontan-bakteriellen Peritonitis und weiteren bakteriellen Infektionen assoziiert [15]. Auch bei Patienten mit Aszites, GI-Blutung oder HE kann ein Dekompensationsereignis im Krankheitsverlauf weitere solche Ereignisse nach sich ziehen [16]. Sobald Patienten mit Zirrhose die erste AD-Episode entwickeln, fällt das mittlere Überleben dramatisch von 12 auf weniger als 2 Jahre ab [15]. Die Auslöser sind maßgeblich bakterielle Infektionen, die schwere alkoholische Hepatitis, GIBlutung mit Schock und die toxische Enzephalopathie. Sie werden im Folgenden besprochen.

\section{Nachgewiesene bakterielle und multiresistente Infektionen}

Bei $43 \%$ der Patienten mit AD ist ein Auslöser identifizierbar. Hier sind wiederum die nachgewiesenen Infektionen (definiert als bakterielle Infektionen mit positiver Erregerkultur) mit 58 \% der Fälle führend [4, 17]. Die Prävalenz multiresistenter Erreger (MRE) nimmt weltweit zu, wobei nach heutigem Stand die multiresistenten gramnegativen Erreger (MRGN) als größte Bedrohung angesehen werden [18].

\section{》) Patienten mit Leberzirrhose und MRE sind kränker als Patienten ohne MRE}

Patienten mit Leberzirrhose und MRE sind kränker als Patienten ohne MRE, was durch Model-of-end-stage-liverdisease(MEL)- und ACLF-Scores quantifizierbar ist, und bei der Entwicklung des ACLF scheinen MRE-Infektionen eine dominante Rolle $\mathrm{zu}$ spielen [16, 19]. Sie sind schwieriger zu behandeln und empirische Therapien schlagen signifikant häufiger fehl [4]. Folglich tritt bei Infektion durch MRE das ACLF signifikant häufiger auf [17, 20, 21]. Im Beitrag von A. Queck, P. Ferstl, T. Bruns, J. Trebicka in diesem Heft wird detail- 
liert auf die bakteriellen Infektionen bei Leberzirrhose und $\mathrm{AD}$ eingegangen.

\section{Schwere alkoholische Hepatitis}

Ungefähr einem Drittel der AD-Fälle geht eine schwere alkoholische Hepatitis (SAH) voraus [6]. Der klinische Verlauf und die Prognose sind dabei ähnlich wie bei Patienten mit nachgewiesenen Infektionen. Auch bei diesen Patienten liegt eine massive Immundysfunktion vor [9]. Weiterhin stört Alkoholkonsum die intestinale Mikroflora und den Gallesäurestoffwechsel und hat durch Beeinflussung der Darm-Hirn-Achse darüber hinaus auch einen indirekten Einfluss auf die kognitive Funktion.

\section{Toxische Enzephalopathie}

Die PREDICT-Studie zeigte, dass neurotoxische Medikamente eine $\mathrm{AD}$ und im weiteren Verlauf ein ACLF auslösen konnten. Dies war nur bei Opioiden und Benzodiazepinen der Fall, während bei Patienten mit hepatotoxischen und nephrotoxischen Medikamenten keine erhöhte AD-Inzidenz beobachtet wurde [4, 6].

\section{Gastrointestinale Blutung mit Schock}

Patienten mit AD und GI-Blutung befinden sich signifikant häufiger in „milderen“ AD-Stadien wie der SDC oder der UDC $[4,6]$. Doch haben Patienten mit ACLF signifikant häufiger eine oder mehrere GI-Blutungen in der Anamnese, als Patienten ohne ACLF [22]. Patienten mit GI-Blutung und Kreislaufversagen sind massiv gefährdet, ein ACLF zu entwickeln [4]. Weiterhin verdoppelt sich die Reblutungsrate in ACLF-Patienten mit Blutungsanamnese, sodass diese ein sehr hohes Sterblichkeitsrisiko aufweisen [22].

\section{Rolle der Auslöseranzahl}

In $57 \%$ der Patienten mit AD kann kein Auslöser gefunden werden [4]. In diesen Fällen wird als Trigger die SI diskutiert [3, 7, 23]. Tatsächlich wird die SI nicht nur in Patienten mit UDC und Prä-ACLF beobachtet, sondern auch in Patienten mit kompensierter Zirrhose oder SDC, die jedoch gleichzeitig eine beginnende PHT aufweisen [3]. Je mehr Auslöser die AD hat, desto ungünstiger entwickeln sich das inflammatorische Profil bzw. die SI und der klinische Verlauf [4].

\section{Verschiedene Verlaufsformen}

Die dekompensierte Zirrhose kann 4 verschiedene Verläufe haben (• Abb. 1; - Abb. 2; [6]). Die SDC ist durch zirrhosetypische Komplikationen, geringe SI sowie die realistische Möglichkeit einer rechtzeitigen Rekompensation gekennzeichnet [11]. Patienten mit SDC müssen üblicherweise nicht aufgrund weiterer Dekompensationsereignisse erneut aufgenommen werden. Organversagen werden bei der SDC selten beobachtet, aber eine Hirn- oder Leberdysfunktion treten in $23 \%$ bzw. $14 \%$ der Patienten auf. In vielen Fällen gehen der SDC bakterielle Infektionen voraus oder begleiten sie. Diese können in der Regel gut behandelt werden, sodass sie ausheilen und der Patient das Stadium der Rekompensation erreicht. Die 1-Jahres-Mortalität bei der SDC beträgt $10 \%$ [6].

\section{\) Patienten mit UDC weisen eine 1-Jahres-Mortalität von 36\% auf}

Die UDC ist mit einer signifikanten PHT assoziiert und weist eine deutlich erhöhte Inzidenz bakterieller Infekte auf, woraus weitere Dekompensationsereignisse resultieren [3, 6]. Nach dem initialen Dekompensationsereignis ist die UDC durch die Notwendigkeit mindestens einer weiteren Krankenhauswiederaufnahme definiert, doch ein ACLF entwickeln diese Patienten nicht. Obwohl Organdysfunktionen bei der UDC öfter auftreten als bei der SD (29\%, 19\% bzw. $16 \%$ für Hirn-, Kreislauf- und Leberdysfunktion), werden Organversagen nur selten beobachtet. Patienten mit UDC weisen eine 1-Jahres-Mortalität von 36\% auf. Maßgeblich ist auch, dass GI-Blutungen deutlich häufiger beobachtet werden als beim Prä-ACLF, was die Rolle der klinisch signifikanten PHT bei der UDC bestätigt [6].

Das Prä-ACLF wird als eine AD-Episode definiert, während oder nach der
Gastroenterologe 2021 · 16:179-185

https://doi.org/10.1007/s11377-021-00520-8

(c) Der/die Autor(en) 2021

P. Ferstl · M. Schulz · J. Trebicka

Akute Dekompensation und akut-auf-chronisches

Leberversagen. Neues zur den

Verlaufsformen der

dekompensierten

Leberzirrhose

\section{Zusammenfassung}

Die akute Dekompensation ist eine rasch eintretende Komplikation der Leberzirrhose. Durch alle Stadien der akuten Dekompensation hinweg weisen die portale Hypertension und systemische Inflammation eine zunehmende Schwere auf. Das akut-aufchronische Leberversagen ist die schwerste Stufe der akuten Dekompensation, und ist durch Organversagen und hohe Mortalität charakterisiert.

Schlüsselwörter

Portale Hypertension . Systemische Inflammation · Auslöser - Score für Organversagen · Intensivtherapie

Acute decompensation and acute-on-chronic liver failure. Update on the stages of decompensated liver cirrhosis

\section{Abstract}

Acute decompensation is defined as complication with an acute onset in endstage liver disease. Throughout the stages of acute decompensation, portal hypertension and systemic inflammation are progressively aggravated. Acute-on-chronic liver failure is the most severe and fatal stage of acute decompensation.

\section{Keywords}

Portal hypertension - Systemic inflammation . Precipitant - Organ failure score - Intensive care

sich ein ACLF entwickelt [6]. Es ist mit signifikanter SI assoziiert und lässt sich somit von der SDC und der UDC unterscheiden. Beim Prä-ACLF tritt die renale Dysfunktion häufiger auf (23\% verglichen mit $7 \%$ bei SDC und UDC). Die 1-Jahres-Mortalität ist mit $67 \%$ ebenfalls deutlich schlechter als bei der SDC und der UDC, und im weiteren Verlauf tritt 


\section{Schwerpunkt}

\begin{tabular}{|l|l|l|l|l|}
\hline & $\begin{array}{l}\text { Stabile } \\
\text { dekompensierte } \\
\text { Zirrhose }\end{array}$ & $\begin{array}{l}\text { Instabile } \\
\text { dekompensierte } \\
\text { Zirrhose }\end{array}$ & $\begin{array}{l}\text { Prä-akut-auf- } \\
\text { chronisches } \\
\text { Leberversagen }\end{array}$ & $\begin{array}{l}\text { Akut-auf-chronisches } \\
\text { Leberversagen }\end{array}$ \\
\hline Kurzfristige Prognose & Rekompensation & Wiederaufnahme & $\begin{array}{l}\text { ACLF-Entwicklung } \\
\text { nach ca. 14 Tagen }\end{array}$ & $\begin{array}{l}\text { Organversagen häufig } \\
\text { Überwachungspflichtig }\end{array}$ \\
\hline Systemische Inflammation & Gering & Mäßig & Schwer & Sehr schwer \\
\hline Komplikationen & Benigner Verlauf & $\begin{array}{l}\text { Komplikationen der } \\
\text { portalen } \\
\text { Hypertension (z.B. } \\
\text { Aszites, Blutungen) }\end{array}$ & $\begin{array}{l}\text { Beginnende } \\
\text { Organversagen }\end{array}$ & $\begin{array}{l}\text { Organversagen, Sepsis, } \\
\text { IMC/ICU }\end{array}$ \\
\hline Mittelfristiger Verlauf & $\begin{array}{l}\text { Keine erneute } \\
\text { Aufnahme }\end{array}$ & $\begin{array}{l}\text { Wiederaufnahme } \\
\text { durch AD }\end{array}$ & Hohe Mortalität & Sehr hohe Mortalität \\
\hline Therapiekonzept & $\begin{array}{l}\text { Wiedervorstellung } \\
\text { in drei Monaten }\end{array}$ & $\begin{array}{l}\text { Therapie der } \\
\text { Komplikationen }\end{array}$ & Evaluation zur LTX & LTX \\
\hline
\end{tabular}

Abb. $2<$ Verschiedene Formen der akuten Dekompensation $(A D)$. ACLF akutauf-chronisches Leberversagen, LTX Lebertransplantation, IMC Intermediate Care, ICU Intensivstation

\begin{tabular}{|c|c|c|c|}
\hline Organsystem & 1 Punkt & 2 Punkte & 3 Punkte \\
\hline Leber & Bilirubin $<6 \mathrm{mg} / \mathrm{dl}$ & $\begin{array}{c}\text { Bilirubin } 6-11,9 \\
\mathrm{mg} / \mathrm{dl}\end{array}$ & Bilirubin $\geq 12 \mathrm{mg} / \mathrm{dl}$ \\
\hline \multirow{2}{*}{ Niere } & Kreatinin $<1,5 \mathrm{mg} / \mathrm{dl}$ & \multirow{2}{*}{ Kreatinin 2-3,4 mg/dl } & Kreatinin $\geq 3,5$ \\
\hline & Kreatinin $1,5-1,9 \mathrm{mg} / \mathrm{dl}$ & & mg/dl oder Dialyse \\
\hline $\begin{array}{l}\text { Gehirn } \\
\text { (West Haven Score) }\end{array}$ & Grad 0 & Grad 1-2 & Grad 1-3 \\
\hline Gerinnung & INR $<2,0$ & INR $2,0-2,4$ & INR $\geq 2,5$ \\
\hline Kreislauf & $M A D \geq 70 \mathrm{mmHg}$ & $\mathrm{MAD}<70 \mathrm{mmHg}$ & Katecholamine \\
\hline \multirow{2}{*}{ Atmung } & $\mathrm{p}_{\mathrm{a}} \mathrm{O}_{2} / \mathrm{F}_{1} \mathrm{O}_{2}>300$ & $\mathrm{p}_{\mathrm{a}} \mathrm{O}_{2} / \mathrm{F}_{1} \mathrm{O}_{2}=201-300$ & $\mathrm{p}_{\mathrm{a}} \mathrm{O}_{2} / \mathrm{F}_{1} \mathrm{O}_{2} \leq 200$ \\
\hline & $\mathrm{S}_{\mathrm{p}} \mathrm{O}_{2} / \mathrm{F}_{1} \mathrm{O}_{2}>357$ & $\mathrm{~S}_{\mathrm{p}} \mathrm{O}_{2} / \mathrm{F}_{1} \mathrm{O}_{2}=215-357$ & $\mathrm{~S}_{\mathrm{p}} \mathrm{O}_{2} / \mathrm{F}_{1} \mathrm{O}_{2} \leq 214$ \\
\hline
\end{tabular}

Abb. $3 \Delta$ Modifiziertes Consortium-organ failure scoring System gemäß European Association for the Study of the Liver (EASL) - Chronic Liver Failure (CLIF). INR International Normalized Ratio, MAD mittlerer arterieller Druck. [5, 24]

als Maximalform der AD das ACLF auf [6].

\section{Akut-auf-chronisches Leberversagen}

\section{Definition und Prognose}

Die europäische (EASL), amerikanische (NACSELD) und asiatische (APASL) hepatologische Fachgesellschaft haben etwas unterschiedliche Definitionen des ACLF vorgeschlagen. Sie unterliegen alle per definitionem der klinischen Charak- terisierung anhand von Organversagen und beinhalten das Vorliegen einer SI [5]. Das ACLF ist eine hochdynamische Entität der $\mathrm{AD}$ und kann innerhalb weniger Tage zum Tod des Patienten führen. Dies beinhaltet auch das klinische Vollbild des Multiorganversagens. Unter allen ACLF-Graden wird das Nierenversagen vor dem Leber- und dem Gerinnungsversagen am häufigsten beobachtet $(56 \%, 44 \%$ und $28 \%$; [1]). Insgesamt umfasst das ACLF 6 Entitäten von Organversagen: Niere, Hirn, Leber, Gerinnung, Kreislauf und/oder Atmung.
Für jedes dieser Organversagen wurden spezifische und exakte Grenzwerte definiert (• Abb. 3). Abhängig von der Anzahl der versagenden Organsysteme ergibt sich der ACLF-Grad von 1 bis 3 (- Abb. 4). Das ACLF ist mit einer 28-Tage-Mortalität von $22 \%$ (ACLF-1) bis zu $77 \%$ (ACLF-3) assoziiert [1]. Die 3-Monats-Mortalität des ACLF liegt, gemittelt für alle Stadien, bei $51 \%$ [1].

\section{Identifizierbare Auslöser und ihre Therapie}

In Fällen mit identifizierbaren Auslösern kommt deren Therapie ein hoher Stellenwert zu. Bei Patienten mit AD, die initial eine inadäquate, d.h. nichtwirksame, antimikrobielle Therapie erhalten, ist das Risiko eines ACLF signifikant erhöht und die 28-Tage-Mortalität steigt - verglichen mit $29 \%$ bei initial adäquater Therapie - auf $54 \%$ an [17]. Da die inadäquate Erstlinienantibiotikatherapie bei nachgewiesener bakterieller Infektion die 90-Tage-Mortalität im ACLF um $>50 \%$ erhöht, wird als Initialtherapie eine breite antimikrobielle Therapie mit Abdeckung der MRE empfohlen [4]. Nach anfänglicher Isolierung des auslösenden Erregers ist eine frühzeitige Therapiedeeskalation entscheidend, um den antimikrobiellen Selektionsdruck und somit das Auftreten von MRE zu minimieren [20]. Daher sollten bei jedem Patienten mit klinisch vermuteter bakterieller und auch fungaler Infektion entsprechende Kulturen und Blutkulturen asserviert werden [15].

Die SAH stellt den zweithäufigsten Auslöser des ACLF dar [4]. Klinische Therapieansätze der SAH sind bislang auf den Einsatz von Kortikosteroiden li- 


\begin{tabular}{lccc}
\hline Patientengruppe & Prävalenz & 28-Tages-Mortalität (\%) & ACLF-Grad \\
\hline Kein OV & 68,3 & 4,4 & Kein ACLF \\
Nicht-renales OV ohne & 9,9 & 6,3 & Kein ACLF \\
renale/zerebrale OD & & & \\
\hline Alleiniges Nierenversagen & 6,7 & 18,6 & ACLF-1 \\
Alleiniges nichtrenales OV mit & 4,2 & 27,8 & ACLF-1 \\
renaler oder zerebraler OD & & & ACLF-2 \\
\hline Zwei OV & 7,5 & 32,0 & ACLF-3 \\
\hline Drei OV & 1,9 & 68,0 & ACLF-3 \\
\hline Vier bis sechs OV & 1,4 & 88,9 & \\
\hline
\end{tabular}

Abb. 4 \ Einteilung nach Organdysfunktion (OD) und Organversagen (OV) in die ACLF-Grade 1 (hellblau), 2 (azurblau) und 3 (blau). ACLF akut-auf-chronisches Leberversagen. [1]

mitiert, die zwar die Kurzzeitmortalität reduzieren, auf den langfristigen Verlauf jedoch keinen Einfluss haben. Zu spezifischeren Therapien der SAH beim ACLF liegen bisher nurvorläufige Daten vor. Bei direkter Leberschädigung durch Hepatitis-B-Reaktivierung kann mittels antiviraler Medikamente ebenfalls spezifisch angesetzt werden.

\section{》) Klinische Therapieansätze der SAH sind auf den Einsatz von Kortikosteroiden limitiert}

Patienten mit ACLF und GI-Blutung profitieren signifikant von einem präemptiven TIPS (pTIPS) verglichen mit ACLF-Patienten und Blutungsanamnese ohne pTIPS (42-Tage-Mortalität: 13,6\% vs. $51,0 \%, 1$-Jahres-Mortalität: $22,7 \%$ vs. $56,5 \%, p=0,002$; [22]). Diese Beobachtungen sind mit älteren Daten vereinbar, nach denen ein pTIPS das Überleben von schwer dekompensierten Patienten mit Varizenblutung und Child-C-Score bis 13 Punkte signifikant verbessert [25-27].

\section{Klinisches Management}

Das klinische Management des ACLF besteht in der Therapie begleitender Organ- versagen. Im Rahmen der intensivmedizinischen Therapie der ACLF-assoziierten Organversagen kann eine kreislaufunterstützende Therapie mit Katecholaminen, bei respiratorischer Verschlechterung eine High-flow-Therapie oder sogar Intubation notwendig sein [28].

Das häufigste Organversagen im ACLF stellt mit etwa $50 \%$ das akute Nierenversagen (acute kidney injury, AKI) dar [1]. Es wird zur Therapie des AKI nach Ausschluss anderer Ursachen und Absetzten aller nephrotoxischen Substanzen eine Plasmaexpansion mit Humanalbumin empfohlen [15]. Bei fehlendem Ansprechen und klinischem Anhalt für ein hepatorenales Syndrom erfolgt die Therapie entsprechend der aktuellen Leitlinie mit Humanalbumin und Terlipressin [29]. Bei refraktärem oder lebensbedrohlichem AKI kann eine Dialyse notwendig werden. Für extrakorporale Leberunterstützungssysteme im ACLF, wie Albumindialyse („molecular adsorbents recirculating system", MARS) und fraktionierte Plasmaseparation und -adsorption (PrometheusSystem, Fresenius Medical Care, Hof an der Saale, Deutschland), konnte in multizentrischen randomisierten Studien bisher kein eindeutiger Überlebensvorteil nachgewiesen werden $[30$,
31]. Interessanterweise ergab eine Subgruppenanalyse der PROMETHEUSStudie einen Überlebensvorteil von Patienten mit einem MELD $>30$. Es muss allerdings einschränkend festgehalten werden, dass diese Studien aus den Jahren 2012 und 2013 vor Konsensus der aktuellen Definition des ACLF gemäß European Association for the Study of the Liver (EASL) - Chronic Liver Failure (CLIF) durchgeführt worden sind. In einer neueren Metaanalyse konnte bei ACLF-Patienten mit einer intensivierten MARS-Therapie ein signifikanter Überlebensvorteil gezeigt werden [32]. Es ist anzunehmen, dass extrakorporale Leberunterstützungssysteme als supportive Verfahren oder zur Überbrückung bis zur Lebertransplantation („,briding to transplant") eine Rolle in der Therapie des ACLF einnehmen werden.

\section{》) Das akute Nierenver- sagen stellt das häufigste Organversagen im ACLF dar}

Im Rahmen der multizentrischen randomisierten GRAFT-Studie sollte der Effekt des granulozytenkoloniestimulierende Faktors (G-CSF) als stammund immunzellmobilisierender Mediator im ACLF untersucht werden [33]. Die GRAFT-Studie musste allerdings vorzeitig geschlossen werden, da sich in der Interimsanalyse keine Verbesserung des ACLF-Schweregrads oder des Überlebens zeigte [34]. Ausgehend von den weitreichenden Implikationen der systemischen Inflammationshypothese werden aktuell verschiedene Therapiestrategien in ACLF-Patienten untersucht.

Es sollte zudem frühzeitig eine Vorstellung an einem Lebertransplantationszentrum erfolgen [15]. Die Lebertransplantation (LTX) stellt die definitive und sicherlich beste Therapie der dekompensierten Leberzirrhose dar. Es gibt bis heute allerdings keinen Konsensus in der spezifischen Priorisierung für ACLF-Patienten. Es konnte gezeigt werden, dass Patienten mit ACLF Grad 3 aufder LTX-Warteliste eine höhere Mortalität aufweisen als Patienten mit akutem Leberversagen. In einer kürzlich veröffentlichten Studie konnte zudem gezeigt werden, dass Pati- 
enten mit ACLF Grad 3 einen deutlichen Überlebensvorteil nach Lebertransplantation haben [35].

\section{Fazit für die Praxis}

- Die akute Dekompensation(AD) ist
die Hauptursache für Morbidität
und Mortalität bei Patienten mit
Leberzirrhose.
- Sie kann verschiedene Verläufe
haben, wobei die stabile dekompen-
sierte Zirrhose die mildeste und das
akut-auf-chronische Leberversagen
(ACLF) die schwerwiegendste und
tödlichste Form sind.
Die 3-Monats-Mortalität des ACLF
liegt bei 51\%. In Abwesenheit von
Organversagen bestimmen das
Ausmaß der portalen Hypertension
und systemische Inflammation den
Krankheitsverlauf.
Wissenschaftliche Anstrengungen
sollten sich u.a. darauf richten, die
klinische Versorgung der AD zu stra-
tifizieren, zu individualisieren und
standardmäßige Therapieregime für
häufig beobachtete AD-Konstellatio-
nen zu etablieren.

\section{Korrespondenzadresse}

Prof. Dr. med. J. Trebicka, PhD

Translationale Hepatologie, Klinik für Innere Medizin I, Goethe-Universität Frankfurt Theodor-Stern-Kai-7, 60590 Frankfurt am Main, Deutschland

jonel.trebicka@kgu.de

\section{Einhaltung ethischer Richtlinien}

Interessenkonflikt. P. Ferstl, M. Schulz und J. Trebicka geben an, dass kein Interessenkonflikt besteht.

Für diesen Beitrag wurden von den Autoren keine Studien an Menschen oder Tieren durchgeführt. Für die aufgeführten Studien gelten die jeweils dort angegebenen ethischen Richtlinien.

Open Access. Dieser Artikel wird unter der Creative Commons Namensnennung 4.0 International Lizenz veröffentlicht, welche die Nutzung, Vervielfältigung, Bearbeitung, Verbreitung und Wiedergabe in jeglichem Medium und Format erlaubt, sofern Sie den/die ursprünglichen Autor(en) und die Quelle ordnungsgemäß nennen, einen Link zur Creative Commons Lizenz beifügen und angeben, ob Änderungen vorgenommen wurden.
Die in diesem Artikel enthaltenen Bilder und sonstiges Drittmaterial unterliegen ebenfalls der genannten Creative Commons Lizenz, sofern sich aus der Abbildungslegende nichts anderes ergibt. Sofern das betreffende Material nicht unter der genannten Creative Commons Lizenz steht und die betreffende Handlung nicht nach gesetzlichen Vorschriften erlaubt ist, ist für die oben aufgeführten Weiterverwendungen des Materials die Einwilligung des jeweiligen Rechteinhabers einzuholen.

Weitere Details zur Lizenz entnehmen Sie bitte der Lizenzinformation auf http://creativecommons.org/ licenses/by/4.0/deed.de.

\section{Literatur}

1. Moreau R, Jalan R, Gines P et al (2013) Acuteon-chronic liver failure is a distinct syndrome that develops in patients with acute decompensation of cirrhosis. Gastroenterology 144(7):1426-1437.e1-9. https://doi.org/10.1053/ j.gastro.2013.02.042

2. Murray CJ, Barber RM, Foreman KJ et al (2015) Global, regional, and national disability-adjusted life years (DALYs) for 306 diseases and injuries and healthy life expectancy (HALE) for 188 countries, 1990-2013: quantifying the epidemiological transition. Lancet 386(10009):2145-2191. https:// doi.org/10.1016/S0140-6736(15)61340-X

3. Trebicka J, Amoros A, Pitarch C et al (2019) Addressing profiles of systemic inflammation across the different clinical phenotypes of acutely decompensated cirrhosis. Front Immunol 10:476. https://doi.org/10.3389/fimmu.2019.00476

4. Trebicka J, Fernandez J, PappMetal (2020) PREDICT identifies precipitating events associated with the clinical course of acutely decompensated cirrhosis. J Hepatol. https://doi.org/10.1016/j.jhep.2020.11. 019

5. Arroyo V, Moreau R, Jalan R (2020) Acute-on-chronic liver failure. N Engl J Med 382(22):2137-2145. https://doi.org/10.1056/NEJMra1914900

6. Trebicka J, Fernandez J, Papp M et al (2020) The PREDICT study uncovers three clinical courses of acutely decompensated cirrhosis that have distinct pathophysiology. J Hepatol. https://doi.org/10. 1016/j.jhep.2020.06.013

7. Schierwagen R, Alvarez-Silva C, Madsen MSA et al (2019) Circulating microbiome in blood of different circulatory compartments. Gut 68(3):578-580. https://doi.org/10.1136/gutjnl-2018-316227

8. Berres ML, Asmacher S, Lehmann J et al (2015) CXCL9 is a prognostic marker in patients with liver cirrhosis receiving transjugular intrahepatic portosystemic shunt. J Hepatol 62(2):332-339. https://doi.org/10.1016/j.jhep.2014.09.032

9. Trebicka J, Bork P, Krag A, Arumugam M (2020) Utilizing the gut microbiome in decompensated cirrhosis and acute-on-chronic liver failure. Nat Rev Gastroenterol Hepatol. https://doi.org/10.1038/ s41575-020-00376-3

10. Clària J, Stauber RE, Coenraad MJ et al (2016) Systemic inflammation in decompensated cirrhosis: characterization and role in acute-on-chronic live failure. Hepatology 64(4):1249-1264. https://doi. org/10.1002/hep.28740

11. Monteiro S, Grandt J, Uschner FE et al (2020) Differential inflammasome activation predisposes to acute-on-chronic liver failure in human and experimental cirrhosis with and without previous decompensation. Gut. https://doi.org/10.1136/ gutjnl-2019-320170
12. López-Vicario $C$, Checa A, Urdangarin A et al (2020) Targeted lipidomics reveals extensive changes in circulating lipid mediators in patients with acutely decompensated cirrhosis. J Hepatol 73(4):817-828. https://doi.org/10.1016/j.jhep. 2020.03.046

13. Moreau R, Clària J, Aguilar F et al (2020) Blood metabolomics uncovers inflammation-associated mitochondrial dysfunction as a potential mechanism underlying ACLF. J Hepatol 72(4):688-701. https://doi.org/10.1016/j.jhep.2019.11.009

14. Laleman W, Claria J, Van der Merwe S, Moreau R, TrebickaJ(2018)Systemicinflammationandacuteon-chronic liver failure: too much, not enough. Can J Gastroenterol Hepatol 2018:1027152. https:// doi.org/10.1155/2018/1027152

15. Angeli P, Bernardi M, Villanueva C et al (2018) EASL clinical practice guidelines for the management of patients with decompensated cirrhosis. J Hepatol 69(2):406-460. https://doi.org/10.1016/j.jhep. 2018.03.024

16. Wong F, Piano S, Singh V et al (2020) Clinical features and evolution of bacterial infectionrelated acute-on-chronic liver failure. J Hepatol. https://doi.org/10.1016/j.jhep.2020.07.046

17. Fernández J, Acevedo J, Wiest R et al (2018) Bacterial and fungal infections in acute-on-chronic liverfailure: prevalence, characteristics and impact on prognosis. Gut 67(10):1870-1880. https://doi. org/10.1136/gutjnl-2017-314240

18. Piano S, Singh V, Caraceni P et al (2019) Epidemiology and effects of bacterial infections in patients with cirrhosis worldwide. Gastroenterology 156(5):1368-1380.e10. https://doi.org/10. 1053/j.gastro.2018.12.005

19. Ferstl PG, Filmann N, Brandt C et al (2017) The impact of carbapenem resistance on clinical deterioration and mortality in patients with liver disease. Liver Int. https://doi.org/10.1111/liv. 13438

20. Ferstl PG, Müller M, Filmann N et al (2018) Noninvasive screening identifies patients at risk for spontaneous bacterial peritonitis caused by multidrug-resistant organisms. Infect Drug Resist 11:2047-2061. https://doi.org/10.2147/ IDR.S172587

21. Fernández J, Prado V, Trebicka J et al (2019) Multidrug-resistant bacterial infections in patients with decompensated cirrhosis and with acuteon-chronic liver failure in Europe. J Hepatol 70(3):398-411. https://doi.org/10.1016/j.jhep. 2018.10.027

22. Trebicka J, Gu W, Ibáñez-Samaniego L et al (2020) Rebleeding and mortality risk are increased by ACLF but reduced by pre-emptive TIPS. J Hepatol 73(5):1082-1091. https://doi.org/10.1016/j.jhep. 2020.04.024

23. Fernández J, Clària J,Amorós A et al (2019) Effects of albumin treatment on systemic and portal hemodynamics and systemic inflammation in patients with decompensated cirrhosis. Gastroenterology 157(1):149-162. https://doi.org/10.1053/j.gastro. 2019.03.021

24. Jalan R, Saliba F, Pavesi Metal (2014) Development and validation of a prognostic score to predict mortality in patients with acute-on-chronic liver failure. J Hepatol 61(5):1038-1047. https://doi. org/10.1016/j.jhep.2014.06.012

25. Kumar R, Kerbert AJC, Sheikh MF et al (2020) Determinants of mortality in patients with cirrhosis and uncontrolled variceal bleeding. J Hepatol. https://doi.org/10.1016/j.jhep.2020.06.010

26. Gerbes A, Labenz J, Appenrodt B et al (2019) Aktualisierte S2k-Leitlinie der Deutschen Gesell- 
schaft für Gastroenterologie, Verdauungs- und Stoffwechselkrankheiten (DGVS) „Komplikationen der Leberzirrhose".Z Zastroenterol 57:611-680

27. de Franchis R, Faculty BV (2015) Expanding consensus in portal hypertension: report of the Baveno $\mathrm{VI}$ consensus workshop: stratifying risk and individualizing care for portal hypertension. J Hepatol 63(3):743-752. https://doi.org/10.1016/ j.jhep.2015.05.022

28. Levesque E, Saliba F, Ichaï P, Samuel D (2014) Outcome of patients with cirrhosis requiring mechanical ventilation in ICU. J Hepatol 60(3):570-578. https://doi.org/10.1016/j.jhep.2013.11.012

29. Piano S, Schmidt HH, Ariza $X$ et al (2018) Association between grade of acute on chronic liver failure and response to terlipressin and albumin in patients with hepatorenal syndrome. Clin Gastroenterol Hepatol 16(11):1792-1800.e3. https://doi.org/10.1016/j.cgh.2018.01.035

30. Kribben A, Gerken G, Haag S et al (2012) Effects of fractionated plasma separation and adsorption on survival in patients with acute-on-chronic liver failure. Gastroenterology 142(4):782-789.e3. https://doi.org/10.1053/j.gastro.2011.12.056

31. Bañares R, Nevens F, Larsen FS et al (2013) Extracorporeal albumin dialysis with the molecular adsorbent recirculating system in acute-onchronic liver failure: the RELIEF trial. Hepatology 57(3):1153-1162. https://doi.org/10.1002/hep. 26185

32. Bañares $R$, Ibáñez-Samaniego $L$, Torner JM et al (2019) Meta-analysis of individual patient data of albumin dialysis in acute-on-chronic liver failure: focus on treatment intensity. Therap Adv Gastroenterol 12:1756284819879565

33. Cornelius E (2020) The liver meeting 2019. Lancet Gastroenterol Hepatol 5(2):112

34. Arroyo V, Moreau R, Jalan $R$, Ginès $P$, EASL-CLIF Consortium CANONIC Study (2015) Acute-onchronic liver failure: a new syndrome that will reclassify cirrhosis. J Hepatol 62(1):S131-43. https:// doi.org/10.1016/j.jhep.2014.11.045

35. Trebicka J, Sundaram V, Moreau R, Jalan R, Arroyo V (2020) Liver transplantation for acute-on-chronic liver failure: science or fiction? Liver Transpl 26(7):906-915. https://doi.org/10.1002/lt.25788

\section{Hepato Update \\ Livestream: \\ Innovativ und flexibel}

11. und 12. Juni aus Mainz

Umfangreiches Update-Wissen in

schwierigen Zeiten: Erstmals können sich interessierte Ärztinnen und Ärzte für das komplette Hepatologie-Update-Seminar zum innovativen Livestream anmelden. Seien Sie LIVE dabei, bequem von zu Hause oder aus der Klinik! Die Interaktivität wird durch einen Livechat ermöglicht und die Zertifizierung ist beantragt, sodass Sie auch bei virtueller Teilnahme Ihre wichtigen CME-Punkte erhalten.

Das Update-Konzept: Die wichtigsten Neuerungen des vergangenen Jahres aus der Hepatologie und hepatobiliären Chirurgie werden unter der wissenschaftlichen Leitung von Prof. Dr. Thomas Berg (Leipzig) und Prof. Dr. Sven Jonas (Nürnberg) kritisch selektiert, analysiert und zusammengefasst. Die Relevanz für Ihren Klinik- und Praxisalltag steht dabei im Vordergrund und der ausführlichen Diskussion über den Livechat mit den Referierenden wird viel Raum gegeben.

In diesem Jahr werden neben den Kerngebieten der Hepatologie die Hot Topics » Infektiologie und SARS-CoV-2« und »Grenzen der Lebertransplantation« vorgestellt.

Zum Gesamtpaket der Teilnahme gehören neben den umfangreichen Seminarunterlagen als eBook der Download aller Vortragspräsentationen und die Vorträge im Nachgang als Video-on-Demand auf der Videoplattform »streamed-up.com«.

Weitere Informationen zu Programm, Referierenden und zur Anmeldung: www.hepato-update.com

Veranstalter:

med update $\mathrm{GmbH}$

Hagenauer Straße 53

65203 Wiesbaden 